\section{Prediction of P-center location from the distribution of energy in the amplitude envelope: II}

\author{
PETER HOWELL \\ University College London \\ London, England
}

Although many of the points discussed by Fowler, Whalen, and Cooper (1988) are disputable, due to space restrictions my comments here are addressed to empirical issues raised in Fowler et al.'s reply. In particular, I will assess their comments concerning my formulas and their experiment.

In their reply to my commentary (Howell, 1988), Fowler et al. (1988) claim that my formula predicts that a stimulus with a long rise has an earlier $\mathrm{P}$ center than one with a short rise, whereas the data in Howell (1984) show the reverse. Fowler et al. estimate the $\mathrm{P}$ centers to occur at 138.9 and $200.2 \mathrm{msec}$ for the sounds with the long and short ramps, respectively. The values they report are for $\mathbf{P}$ centers measured not from stimulus onset but when the $x$-axis origin is at the point where the initial section and rectangle meet (the origin was shown in this position in the figures to make the derivation clear). If the model is applied as described in my earlier comment (Howell, 1988), the stimulus with the short ramp is predicted to have a $P$ center $240.2 \mathrm{msec}$ from the start of the sound and the stimulus with the long ramp $(120 \mathrm{msec})$ to have one $258.9 \mathrm{msec}$ from the start. Fowler et al.'s calculations are incorrect; the formula predicts that the stimulus with the gradual rise has a later $\mathrm{P}$ center, consistent with results in Howell (1984).

The new peak-clipping experiment is not a satisfactory test between the hypotheses of Howell (1988) and Cooper, Whalen, and Fowler (1986) in view of problems with their

The author's mailing address is Department of Psychology, University College London, Gower Street, London WCIE 6BT, England. stimuli and the fact that the centers of gravity they calculate are not the centers of gravity of the envelopes. First, the stimuli are not, in fact, peak-clipped. Instead, specified portions of their waveforms have been scaled up to use the full range on the D-to-A converters. This is obviously so in their sa $\mathrm{s}_{\mathrm{s}}$ stimulus. The process of scaling up parts of a sound is different from that of peak-clipping them, and causes spectral distortion by raising the noise floor. Second, the /s/ sound in Fowler et al.'s Figure 2 is periodic, which it should not be.

Finally, the center of gravity they calculate is not the same as the center of gravity of the amplitude envelope. The amplitude envelope can be obtained by low-pass filtering the output of a full-wave linear rectifier (Howell, 1984 , referred readers to Fant, 1959, for details). Working directly from digital waveforms, as Fowler et al. (1988) have done, omits the low-pass filtering operation. Thus, it is not clear whether the scaling of the center of gravities is anything like what would arise if the center of gravity of the syllables had been calculated from envelopes as intended by Howell (1987).

\section{REFERENCES}

Cooper, A. M., Whalen, D. H., \& Fowler, C. A. (1986). P-centers are unaffected by phonetic categorization. Perception \& Psychophysics, 39, 187-196.

FANT, G. M. C. (1959). Acoustic analysis and synthesis of speech with applications to Swedish. Ericsson Technics, 1, 1-105.

Fowler, C. A., Whalen, D. H., \& CoOper, A. M. (1988). Perceived timing is produced timing: A reply to Howell. Perception \& Psychophysics, 43, 94-98.

Howell, P. (1984). An acoustic determinant of perceived and produced anisochrony. In M. P. R. Van den Broecke \& A. Cohen (Eds.), Proceedings of the 10th International Congress of Phonetic Sciences (pp. 429-433). Dordrecht, Holland: Foris.

Howell, P. (1988). Prediction of P-center location from the distribution of energy in the amplitude envelope: I. Perception \& Psychophysics, 43, 90-93.

(Manuscript received June 16, 1987; revision accepted for publication June $24,1987$. ) 